\title{
A constitutive law for snow taking into account the compressibility
}

\author{
Aloke MiSHRA,${ }^{1}$ Puneet MAHAJAN ${ }^{2}$ \\ ${ }^{1}$ Snow and Avalanche Study Establishment, Manali 175 103, Himachal Pradesh, India \\ ${ }^{2}$ Indian Institute of Technology, Delhi, Hauz Khas, New Delhi 110016, India \\ E-mail:mahajan@am.iitd.ernet.in
}

\begin{abstract}
A constitutive law for snow derived from a complementary power potential is proposed. The total deformation of snow is divided into elastic and creep parts. A hereditary integral using Norton's power law is employed to describe primary creep. The concept of effective stress, which takes compressibility of snow into account, is used to calculate creep deformation. The hereditary integral is approximated by a non-linear spring-dashpot model. Results from uniaxial compression experiments (stress range 15$45 \mathrm{kPa}$ ) on sieved snow of density range $180-470 \mathrm{~kg} \mathrm{~m}^{-3}$ were used to determine the constants appearing in the constitutive equation. The response of snow to constant strain rate $\left(7.4 \times 10^{-6} \mathrm{~s}^{-1}\right.$ to $\left.2.2 \times 10^{-5} \mathrm{~s}^{-1}\right)$ under bilaterally confined conditions was found with an iterative scheme employing the proposed constitutive law. The simulated results agree well with the measured axial stresses and volumetric changes.
\end{abstract}

\section{INTRODUCTION}

Snow is a non-linear compressible viscoelastic material. Modelling of the continuously changing stress regime in creeping snowpack on an avalanche slope requires a multiaxial constitutive law. Prior to 1970, the equation for a fourparameter viscoelastic fluid with linear elements was used as the most general constitutive relationship (Mellor, 1964). Salm (1967) investigated multiaxial behaviour of snow using a constitutive law similar to Hooke's law, with strain rate in place of strain. Probably the first comprehensive non-linear constitutive relation was proposed by Salm (1971). An approach similar to the stress-strain relationship for a Green-Rivlin material was used by Brown and others (1973) and Brown (1976) to describe the non-linear deformation behaviour of snow. Salm (1977) developed a constitutive equation for creeping snow in a quasi-stationary state by the principle of maximum entropy production.

In this paper, we extend the phenomenological approach of Szyszkowski and Glockner (1986) for incompressible ice to compressible snow. This is achieved by defining an effective viscoelastic stress, which takes into account the effect of compressibility. The deformation of snow is divided into elastic, primary creep and secondary creep. A nonlinear hereditary integral is used to describe the primary creep. Secondary creep strain is approximated by a generalized Norton's power law. In the phenomenological approach used here, the constants appearing in the constitutive law are determined from macroscopic observations without considering microstructure or its evolution. Instead, these constants are related to the relative density. Finally, the proposed constitutive law is applied to triaxial test data to determine how well it approximates this behaviour. The snow here is assumed to be isotropic and non-age-hardening. Both these assumptions seem reasonable for the duration and environmental conditions of the laboratory experiment. Snow samples prepared and tested under laboratory conditions show comparatively less scatter in deformation behaviour and properties, thereby facilitating analytical modelling. Hence, results of cold-laboratory experiments on sieved snow samples were used both to determine the constants and to validate the law.

\section{MULTIAXIAL CONSTITUTIVE LAW}

For snow we propose the existence of a complementary power potential $W$ such that

$$
D_{i j}=D_{i j}^{\mathrm{e}}+D_{i j}^{\mathrm{c}}=\frac{\mathrm{d} W}{\mathrm{~d} \sigma_{i j}},
$$

where $D_{i j}$ is the rate-of-deformation tensor which is divided into elastic and creep parts $D_{i j}^{\mathrm{e}}$ and $D_{i j}^{\mathrm{c}}$. The complementary power potential is expressed as a function of the first and second invariant of stress tensor $\sigma_{i j}$ and consists of three terms:

$$
\begin{aligned}
W= & \frac{\mathrm{d}}{\mathrm{d} t}\left(\frac{\sigma_{\mathrm{m}}^{2}}{2 K}+\frac{S^{2}}{6 G}\right)+A_{1} \frac{\mathrm{d}}{\mathrm{d} t} \int_{0}^{t} F[P(\sigma)] j(t-\tau) \mathrm{d} \tau \\
& +A_{2} \int_{0}^{t} F[P(\sigma)] .
\end{aligned}
$$

The first term represents linear elastic strain energy, and the second is recoverable viscoelastic potential, while the third term signifies permanent deformation. In Equation (2), $K$, $G$ and $\sigma_{\mathrm{m}}$ denote the elastic bulk modulus, shear modulus and hydrostatic stress respectively. The second invariant of deviatoric-stress tensor $s_{p q}$ is depicted as

$$
S^{2}=(3 / 2) s_{p q} s_{p q} \quad s_{p q}=\sigma_{p q}-\frac{1}{3} \sigma_{k k} \delta_{i j}
$$




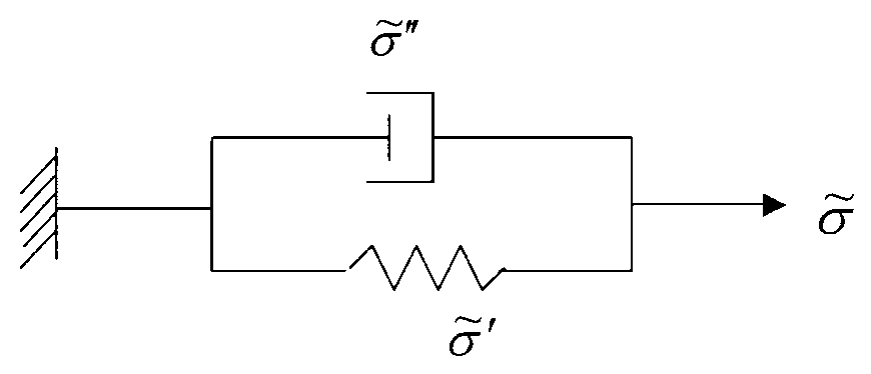

Fig. 1. A non-linear spring-dashpot model for primary creep.

The function $F[P(\sigma)]$ is defined as

$$
\begin{aligned}
& F(P)=\frac{B P^{n+1}}{n+1} \quad P^{2}=\frac{3 c}{2} s_{p q} s_{p q}+f(\operatorname{tr} \sigma)^{2} \\
D_{i j}^{\mathrm{c}}= & A_{1} \frac{\mathrm{d}}{\mathrm{d} t} \int_{0}^{t} B P^{n-1}\left(\frac{3 c}{2} s_{i j}+f \sigma_{k k} \delta_{i j}\right) j(t-\tau) \mathrm{d} \tau \\
& +A_{2} B P^{n-1}\left(\frac{3 c}{2} s_{i j}+f \sigma_{k k} \delta_{i j}\right)
\end{aligned}
$$

where $[P(\sigma)]$ is the effective stress (Chenot and others, 1990).

Using Equations (1-4), we have

$$
D_{i j}^{\mathrm{e}}=\frac{1}{E}\left[(1+\mu) \dot{\sigma}_{i j}-3 \dot{\sigma}_{\mathrm{m}} \delta_{i j}\right]
$$

where $\mu$ is the Poisson's ratio.

Defining

$$
\tilde{\sigma}_{i j}^{n}=P^{n-1}\left[\frac{3 c}{2} s_{i j}+f \sigma_{k k} \delta_{i j}\right],
$$

and replacing $A_{1} B$ and $A_{2} B$ by $1 / \nu_{1}$ and $1 / \nu_{2}$ respectively, the creep deformation rate can be written as

$$
D_{i j}^{\mathrm{c}}=D_{i j}^{\mathrm{p}}+D_{i j}^{\mathrm{s}}=\frac{1}{\nu_{1}} \frac{\mathrm{d}}{\mathrm{d} t} \int_{0}^{t} \tilde{\sigma}_{i j}^{n} j(t-\tau) \mathrm{d} \tau+\frac{1}{\nu_{2}} \tilde{\sigma}_{i j}^{n} .
$$

$D_{i j}^{\mathrm{p}}$ and $D_{i j}^{\mathrm{s}}$ are the primary and secondary creep deformation rates. The function $j(t)$ is normalized such that $j(0)=1$ and $j(t) \rightarrow 0$, as $t \rightarrow \infty$; this implies that area $(J)$ under the $j$ vs time curve is finite, i.e.

$$
J(t \rightarrow \infty)=\int_{0}^{t \rightarrow \infty} j(\tau) \mathrm{d} \tau=J_{\infty}
$$

A non-linear spring-dashpot model (Fig. 1) is used to

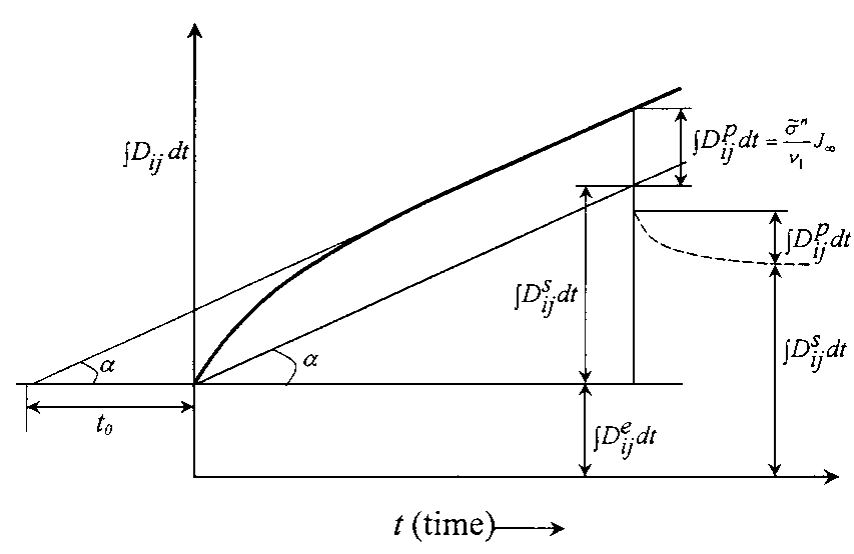

Fig. 2. Concept of $t_{0}$ shown graphically on a typical creep curve.

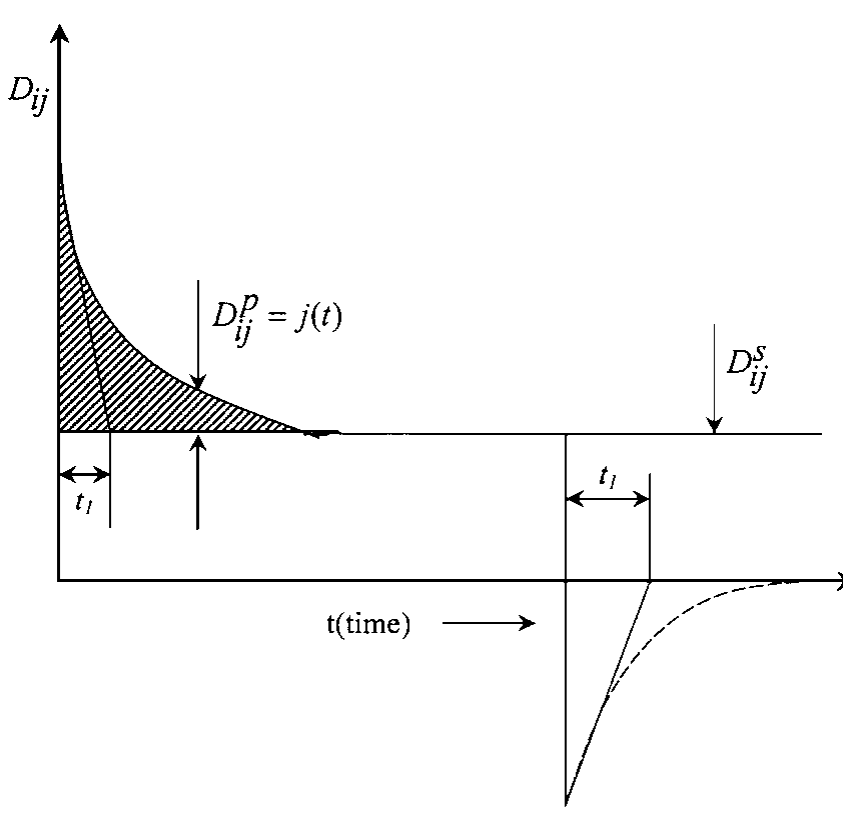

Fig. 3. Concept of $t_{1}$ shown on a strain-rate vs time plot.

represent the hereditary integral $j(t)$ (Szyszkowski and others, 1986), so that

$$
\begin{aligned}
\left(\tilde{\sigma}_{i j}{ }^{\prime \prime}\right)^{n} & =\nu_{1} D_{i j}^{\mathrm{p}}, \\
\left(\tilde{\sigma}_{i j}{ }^{\prime}{ }^{n}+\alpha\left(\tilde{\sigma}_{i j}\right)^{n-1} \tilde{\sigma}_{i j}{ }^{\prime}\right. & =E_{1} \int_{0}^{t} D_{i j}^{\mathrm{p}} \mathrm{d} t, \\
\tilde{\sigma}_{i j}{ }^{\prime}+\tilde{\sigma}_{i j}{ }^{\prime \prime} & =\tilde{\sigma}_{i j}
\end{aligned}
$$

( $\alpha$ is introduced so that $E_{1} \neq 0$ at $\tilde{\sigma}=0$ ).

The postulated constitutive law expressed as Equation (8) implies certain invariant features for creep curves for various constant stress levels. It can be shown that

$$
\int_{0}^{\infty}\left(D_{i j}-D_{i j}^{\mathrm{s}}\right) \mathrm{d} t=\frac{\tilde{\sigma}_{i j}^{n}}{\nu_{1}} J_{\infty}=t_{0} \tan \alpha=t_{0} \frac{\tilde{\sigma}_{i j}^{n}}{\nu_{2}} .
$$

We thus obtain a stress-independent parameter $t_{0}$ (see Fig. 2):

$$
t_{0}=\frac{\nu_{2}}{\nu_{1}} J_{\infty}
$$

From Equation (8), we obtain

$$
\frac{\mathrm{d} D_{i j}}{\mathrm{~d} t}=\left.\frac{\tilde{\sigma}_{i j}^{n}}{\nu_{1}} \frac{\mathrm{d} j}{\mathrm{~d} t}\right|_{t=0}=-\cot \gamma=-\frac{\tilde{\sigma}_{i j}^{n}}{\nu_{1} t_{1}} .
$$

A second stress-independent parameter, $t_{1}$, can be written as (see Fig. 3)

$$
t_{1}=-\frac{1}{\left.\frac{\mathrm{d} j}{\mathrm{~d} t}\right|_{t=0}} .
$$

When a constant stress is removed at time $t=t^{\prime}$, the reversible portion, amounting to $\left(\tilde{\sigma}_{i j}^{n} / \nu_{1}\right) J\left(t^{\prime}\right)$, should be recovered while the irreversible component, $\left(\tilde{\sigma}_{i j}^{n} / \nu_{2}\right) t^{\prime}$, remains as permanent viscous strain. If the time, $t^{\prime}$, is chosen such that the creep process is in its "steady creep" stage, measurements of the "permanent" $\int D_{i j}^{\mathrm{s}} \mathrm{d} t$ and "recoverable" $\int D_{i j}^{\mathrm{p}} \mathrm{d} t$ viscous strains may also be used in determining the parameter, $t_{0}$, from the relation

$$
t_{0}=\frac{\int D_{i j}^{\mathrm{p}} \mathrm{d} t}{\int D_{i j}^{\mathrm{s}} \mathrm{d} t} t^{\prime}=\frac{\int D_{i j}^{\mathrm{p}} \mathrm{d} t}{\tilde{\sigma}_{i j}^{n}} \nu_{2} .
$$

The memory function $j(t)$ in the proposed equation is approximated with that of the model's $j_{\mathrm{m}}(t)$ shown in Figure 1 and expressed by Equations $(10 \mathrm{a}-\mathrm{c}) \cdot j_{\mathrm{m}}(t)$ is a characteristic function of the model describing the hardening 
process during primary creep stage. For the model, the creep deformation-rate tensor $D_{i j}^{\mathrm{c}}$ can be written as

$$
D_{i j}^{\mathrm{c}}=\frac{\tilde{\sigma}_{i j}^{n}}{\nu_{1}} j_{\mathrm{m}}(t)+\frac{\tilde{\sigma}_{i j}^{n}}{\nu_{2}} .
$$

Comparing the first term with Equation (10a),

$$
\tilde{\sigma}_{i j}{ }^{\prime \prime}(t)=\tilde{\sigma}^{n} \sqrt{j_{\mathrm{m}}(t)}
$$

which, when used with Equations (10b) and (10c) (for spring element), gives

$$
\tilde{\sigma}_{i j}{ }^{\prime}(t)=\tilde{\sigma}\left(1-\sqrt[n]{j_{\mathrm{m}}}(t)\right)
$$

and

$$
\left[1-\sqrt[n]{j_{\mathrm{m}}(t)}\right]^{n}+\alpha\left[1-\sqrt[n]{j_{\mathrm{m}}(t)}\right]=\frac{E_{1} \int D_{i j}^{\mathrm{p}} \mathrm{d} t}{\tilde{\sigma}^{n}} .
$$

From Equation (19), by differentiation, we obtain

$$
\frac{\mathrm{d} j_{\mathrm{m}}(t)}{\mathrm{d} t}=-\frac{E_{1}\left(j_{\mathrm{m}}\right)^{2-\frac{1}{n}}}{\nu_{1}\left[\left(1-\sqrt[n]{j_{\mathrm{m}}}\right)^{n-1}+\frac{\alpha}{n}\right]} .
$$

Substituting Equations (14) and (20) into Equation (10a), one arrives at

$\frac{1}{t_{1}}=\frac{E_{1}}{\nu_{1}} \frac{1}{\delta+\alpha / n}, \quad$ where $\quad \delta=\mid \begin{array}{lll}1 & \text { for } & n=1.0 \\ 0 & \text { for } & n>1.0\end{array}$.

In the model, it may be appreciated that as $t \rightarrow \infty$, $\tilde{\sigma}^{\prime \prime}(t) \rightarrow 0$, while ultimately the total stress applied to the model is taken by the spring. This condition, together with Equations (16) and (10b), leads to

$$
\int_{0}^{\infty} D_{i j}^{\mathrm{p}} \mathrm{d} t=\frac{\tilde{\sigma}^{n}}{\nu_{1}} \int_{0}^{\infty} j_{\mathrm{m}}(t) \mathrm{d} t=(1+\alpha) \frac{\tilde{\sigma}^{n}}{E_{1}}
$$

from which we obtain

$$
\frac{E_{1}}{\nu_{1}} J_{\infty}=1+\alpha
$$

Using Equations (21), (23) and (12), two additional constants, $\alpha$ and $E_{1}$, for the model can be related to the material parameters, $t_{0}$ and $t_{1}$ (for $n>1$ ), by

$$
\begin{aligned}
\alpha & =\frac{\lambda}{1-\lambda} \\
E_{1} & =\frac{\nu_{1}}{t_{1}} \frac{\lambda}{n} \frac{1}{1-\lambda},
\end{aligned}
$$

where

$$
\lambda=\frac{\nu_{2}}{\nu_{1}} \frac{t_{1}}{t_{0}} n
$$

From the above it is evident that the determination of strain in snow requires knowledge of all the constants $\nu_{1}, \nu_{2}, t_{0}, t_{1}$, $E_{1}, \alpha, n, c$ and $f$ appearing in the constitutive law.

The authors realize that the spring-dashpot model for snow may not be very exact. In snow, primary creep occurs largely from deformation of bonds/grains, whereas secondary creep occurs both from deformation of bonds/grains and from sliding of grains after bonds break. If grain sliding is the predominant deformation mechanism, only a fraction of the primary creep strain will be recovered. On unloading, a spring-dashpot model will show full recovery over a period of time, which may not happen in the case of snow.

\section{EXPERIMENTS}

Three sets of experiments were conducted for the present study: (1) Over 60 constant-load unconfined compression creep tests were conducted, results of which were utilized for the determination of all the constants appearing in the developed constitutive law. (2) Results from about 15 constant-load unconfined compression creep experiments were used to validate the determined constants. (3) About 60 constant displacement-rate tests under different confining pressures were conducted. Since the constants are determined from constant-load experiments, the form of the proposed constitutive law will be confirmed if it works for triaxial state of stress under altogether different boundary condition, i.e. constant displacement rate.

\section{Uniaxial tests}

Snow samples collected from the field are stored in a small cold chamber, maintained at $-20^{\circ} \mathrm{C}$. Cylindrical samples (diameter: $0.065 \mathrm{~m}$; height: $0.15 \mathrm{~m}$ ) are prepared by sieving to maintain a uniform grain-size of $0.5 \times 10^{-3} \mathrm{~m}$ to $1.0 \times 10^{-3} \mathrm{~m}$. Tests were performed on a $10 \mathrm{kN}$ Universal Testing Machine. The density range of samples was 180$470 \mathrm{~kg} \mathrm{~m}^{-3}$. No sample was more than 14 days old. All tests were performed for 8 hours at $-10^{\circ} \mathrm{C}$. Lateral strain rate was monitored with a spring-loaded displacement sensor. Stresses applied were in the range $0.015-0.045 \mathrm{MPa}$.

\section{Multiaxial tests}

A triaxial testing machine for snow, developed and installed in the cold room of the Snow and Avalanche Study Establishment (SASE), Manali, India, can conduct constantstrain-rate (CSR) tests under different confining pressures (up to $0.030 \mathrm{MPa}$ ). It is also capable of recording the volumetric change in the sample with the help of a flow-meter attachment. Snow type, sample preparation method and sample dimensions were the same as those used for uniaxial experiments. Strain rates ranging from $7.4 \times 10^{-6} \mathrm{~s}^{-1}$ to $2.2 \times 10^{-5} \mathrm{~s}^{-1}$ were used. Again, samples up to 14 days old were tested at an environment temperature of $-10^{\circ} \mathrm{C}$.

\section{DETERMINATION OF PARAMETERS FROM UNIAXIAL TESTS}

During uniaxial tests, from the instantaneous axial and lateral deformation at $t=0$, the Young's modulus $E$ and Poisson's ratio $\mu$ were determined. Based on these, three values for $E$, namely 4,10 and $17 \mathrm{MPa}$, were used for snowdensity ranges $230-300 \mathrm{~kg} \mathrm{~m}^{-3}, \quad 300-370 \mathrm{~kg} \mathrm{~m}^{-3}$ and $370 \mathrm{~kg} \mathrm{~m}^{-3}$ and above, respectively.

For the uniaxial unconfined creep test, the primary creep rate reduces continuously and acquires a negligible value in $1-2$ hours in a test conducted for 8 hours. With this assumption, for time $t>2$ hours

$$
D_{11}^{\mathrm{c}}=D_{11}^{\mathrm{s}}=\frac{\sigma_{0}^{n}}{\nu_{2}}(c+f)^{\frac{n+1}{2}},
$$

where $\sigma_{0}{ }^{n}$ is applied axial stress.

The following form of $c$ and $f$ was chosen so that the constitutive law reduces to that for incompressible matrix material for relative density, i.e. $\rho_{\text {snow }} / \rho_{\text {ice }}=\gamma=1$ :

$$
c=1+c_{1}(1-\gamma)^{q} ; \quad f=c_{2}(1-\gamma)^{r} .
$$

By substituting Equations (26a) and (26b) into Equation (25),

$$
D_{11}^{\mathrm{s}}=\frac{\tilde{\sigma}_{0}^{n}}{\nu_{2}}\left[1+c_{1}(1-\gamma)^{q}+c_{2}(1-\gamma)^{r}\right]^{\frac{n+1}{2}} .
$$

By performing creep tests on two samples of the same 
Table 1. Values and units of the constants determined

\begin{tabular}{ccc}
\hline Notations for constants & Unit & Average value \\
\hline$C_{1}$ & Dimensionless & 3279 \\
$C_{2}$ & Dimensionless & 7256 \\
$\nu_{2}$ & $(\mathrm{MPa})^{n} \mathrm{~s}$ & $3.9 \times 10^{6}$ \\
$t_{0}$ & $\mathrm{~s}$ & $2.57 \times 10^{4}$ \\
$r$ & Dimensionless & 5.63 \\
$t_{1}$ & $\mathrm{~s}$ & 100 \\
$q$ & Dimensionless & 5.07 \\
$\nu_{1}$ & $(\mathrm{MPa})^{n} \mathrm{~s}$ & $1.8 \times 10^{5}$ \\
$E_{1}$ & $(\mathrm{MPa})^{n}$ & 215.2 \\
$\alpha$ & Dimensionless & 0.1764 \\
$n$ & Dimensionless & 1.48 \\
\hline
\end{tabular}

density, the approximate value of $n$ can be determined from Equation (25).

Writing an equation for $D_{22}^{\mathrm{s}}$ similar to Equation (27), and dividing that by Equation (27),

$$
c_{1}-c_{2}(1-\gamma)^{q-r}\left[\frac{\frac{D_{22}}{D_{11}}+0.5}{1-\frac{D_{22}}{D_{11}}}\right]=0 .
$$

A regression fit, using software developed by D. Hyams (http://www.ebicon.com/ dhyams/cvxpt.html), performed on Equations (28) and (27) gives $c_{1}, c_{2}, q, r$ and $\nu_{2}$. We next determine the constants $\nu_{1}, E_{1}, \alpha$ required in primary creep equations. $\nu_{1}$ is calculated from the deformation rate at $t=0$ from the following equation:

$$
\frac{1}{\nu_{1}}=\frac{\left(D_{11}\right)_{t=0}-\frac{\sigma_{0}{ }^{n}}{\nu_{2}}(c+f)^{\frac{n+1}{2}}}{\sigma_{0}^{n}(c+f)^{\frac{n+1}{2}}} .
$$

The determination of $E_{1}$ and $\alpha$ requires evaluation of $t_{0}$ and $t_{1}$ as shown in Equations $(24 \mathrm{a}-\mathrm{c})$. Table 1 lists the values of various constants appearing in the constitutive equation. The proposed law is now used to predict the deformation behaviour of snow for uniaxial confined compression tests not used in determining the constants. Figures 4 and 5 show the comparison of actual and simulated creep response for low- and high-density snow, respectively. The constitutive law predicts the creep response accurately, though it appears that in a few cases the difference is appreciable (e.g. Fig. 6). Since, the values of $c$ and $f$ have been considered to be initial-density-dependent only, a different microstructure arrangement may significantly alter the snow response.

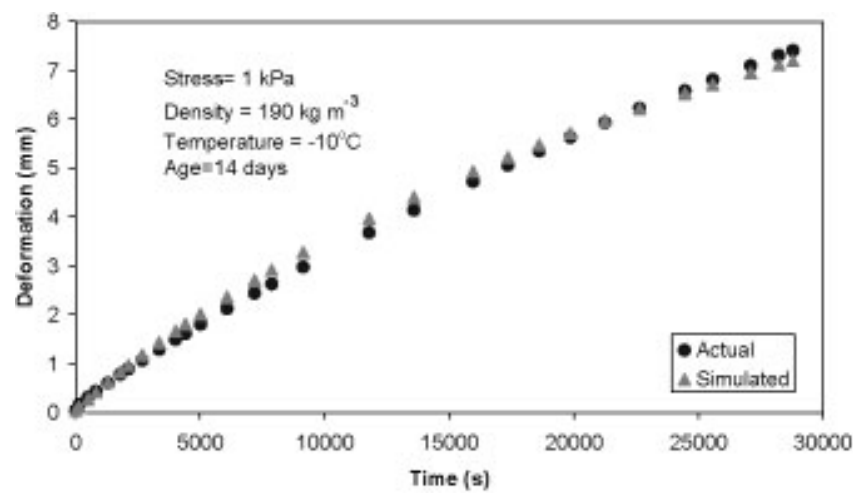

Fig. 4 Comparison of actual and simulated deformation for low-density snow.

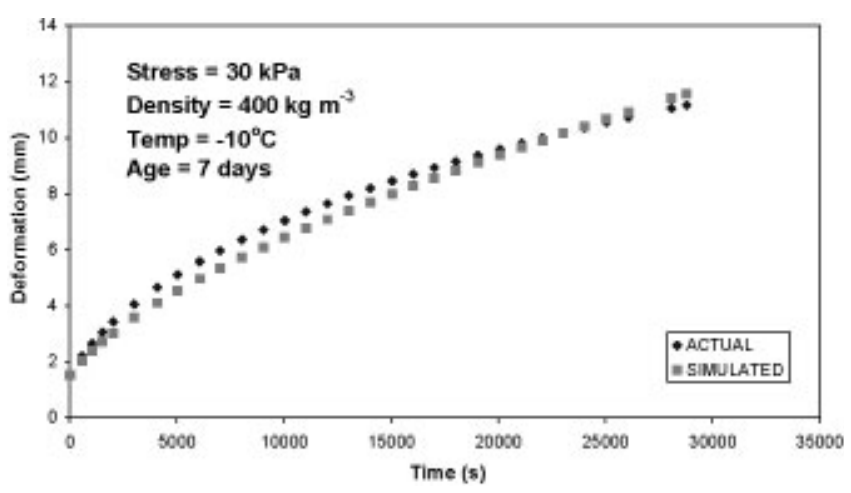

Fig. 5. Comparison of actual and simulated creep deformation for high-density snow.

\section{MULTIAXIAL TESTS}

Validation of the proposed constitutive equation was attempted along similar lines to Desrues and others (1980). To determine the longitudinal stress and volume change, corresponding to the above strain rates and confining pressure, from the model, the following equations, combined with Equation (8), were solved in an iterative manner:

$$
\begin{aligned}
\sigma_{11}= & \frac{E}{(1+\nu)}\left(\frac{1-\nu}{1-2 \nu}\right) \int_{0}^{t}\left(D_{11}-D_{11}^{\mathrm{c}}\right) \mathrm{d} t \\
& +2 \frac{\nu E}{(1+\nu)(1-2 \nu)} \int_{0}^{t}\left(D_{22}-D_{22}^{\mathrm{c}}\right) \mathrm{d} t \\
\sigma_{22}= & \frac{E(1+\nu)}{(1+\nu)(1-2 \nu)} \int_{0}^{t}\left(D_{22}-D_{22}^{\mathrm{c}}\right) \mathrm{d} t \\
& +\frac{\nu E}{(1+\nu)(1-2 \nu)} \int_{0}^{t}\left(D_{11}-D_{11}^{\mathrm{c}}\right) \mathrm{d} t .
\end{aligned}
$$

For the given strain rates, the axial stress developed was in the range $0.02-0.14 \mathrm{MPa}$. Figure 7 shows the comparison of actual and simulated axial stress developed in the sample due to the subjection of the sample to constant strain rate under lateral confining pressure. It may be noted that stress develops more during the first $500 \mathrm{~s}$ than has been predicted. One may expect fast adjustment of grains/bonds during the initial period of strain-rate application for such moderate-density snow. Figure 8 shows a comparison of actual and simulated volume change. The theory simulates the experimental results moderately well.

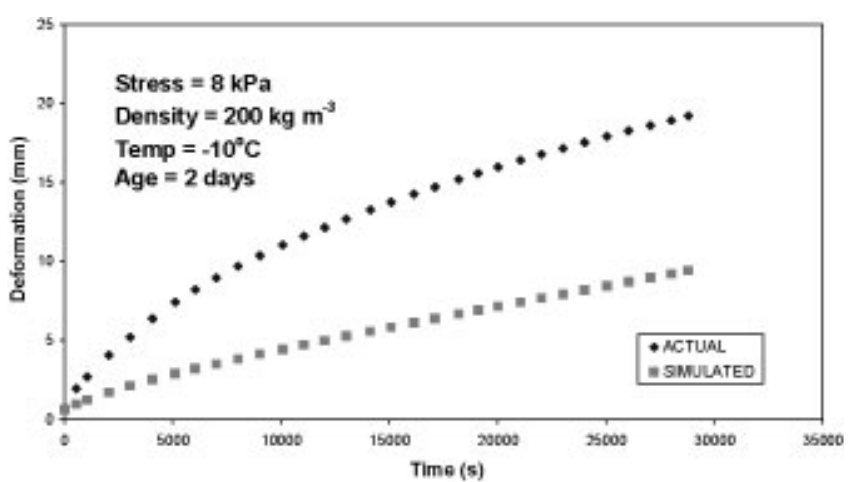

Fig. 6. Comparison of actual and simulated deformation for low-density snow. 


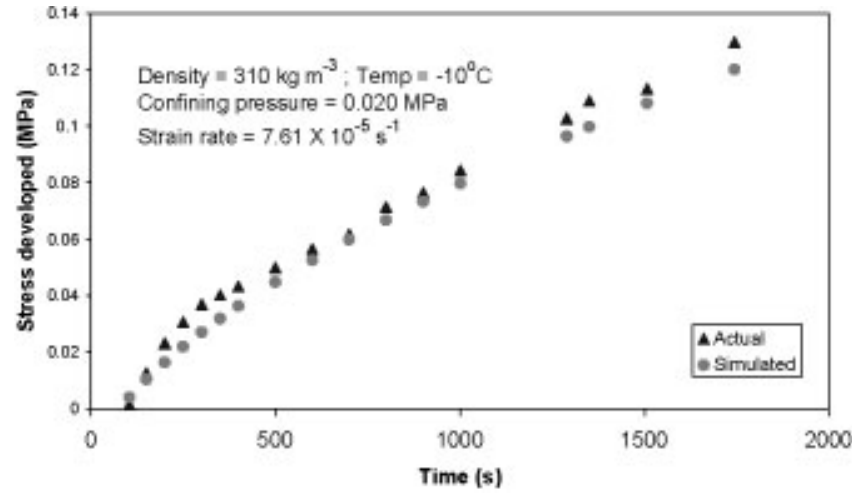

Fig. 7. Simulated developed axial stress, compared with triaxial test result.

\section{CONGLUSION}

A constitutive law has been proposed, and various constants required for it have been determined from creep tests on snow. A comparison of the model predictions with uniaxial tests other than those used to determine constants showed a good match. Also, the generally overlooked primary-stage strain contribution in creep response is predicted satisfactorily. In certain cases, however (e.g. highdensity sample subjected to long-duration curing and lowdensity samples of less age $(<7$ days $))$, the creep deformation predictions were not very accurate. In the model, parameters $c$ and $f$ were introduced as functions of initial density. In future we aim to relate these to a non-dimensional parameter (Sethi and others, 2002) which characterizes snow. Experiments (with microstructure index measured) are in progress to see if these parameters can be made dependent upon the index. In nature, creep occurs over a long time and the snow density shows significant variation. To account for this it is possible to have different forms of $P$ in primary and secondary creep. For example, " $P$ " in primary creep (which becomes insignificant in 1-2 hours) may be taken as a function of initial density whereas it may be taken as a function of current density for secondary creep. Since density changes are not very high in the laboratory, in the proposed constitutive law $P$ is assumed to depend on initial density only.

The triaxial tests were also performed and the constitutive model was used to predict the behaviour. Generally, the model accurately predicts the stresses developed and the volumetric change for bilaterally confined snow samples subjected to axial strain rates. The same theory can be extended to tensile tests by employing the damage factor.

The highlight of this proposed law is its ability to deter-

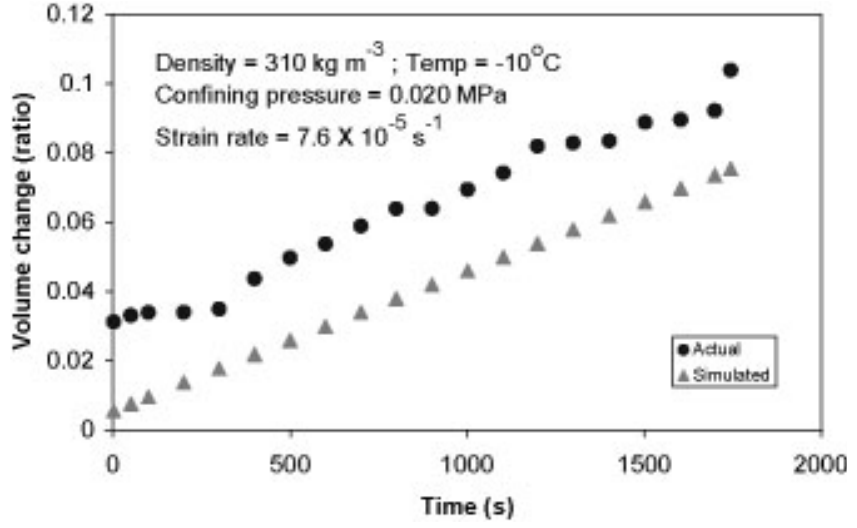

Fig. 8. Simulated volume change is slightly less than the actual one.

mine volumetric change, while a moderate degree of accuracy is obtained in the prediction of volume change.

\section{AGKNOWLEDGEMENT}

The authors wish to express their gratitude for the support rendered by C. Chandel, Scientist "B" of SASE, for the experimentation during this work.

\section{REFERENGES}

Brown, R. L. 1976. A thermodynamic study of materials representable by integral expansions. Int. F. Eng. Sci., 14(11), 1033-1046.

Brown, R. L., T. E. Lang, W. F. St Lawrence and C. C. Bradley. 1973. Failure criterion for snow. 7. Geophys. Res., 78(23), 4950-4958.

Chenot, J.-L., F. Bay and L. Fourment. 1990. Finite element simulation of metal powder forming. Int. F. Numer. Methods Eng., 30(8), 1649-1674.

Desrues, J., F. Darve, E. Flavigny, J. P. Navarre and A. Taillefer. 1980. An incremental formulation of constitutive equations for deposited snow. 7. Glaciol., 25(92), 289-307.

Mellor, M. 1964. Properties of snow. CRREL Monogr. III-Al.

Salm, B. 1967. An attempt to clarify triaxial creep mechanics of snow. In Öura, H., ed. Physics of snow and ice. Vol. 1, Part 2. Sapporo, Hokkaido University. Institute of Low Temperature Science, 857-874.

Salm, B. 1971. On the rheological behaviour of snow under high stresses. Contrib. Inst. Low Temp. Sci., Ser. A, 23, 1-43.

Salm, B. 1977. Eine Stoffgleichung für die Kriechende Verformung von Schnee. (Ph.D. thesis, Eidgenössische Technische Hochschule, Zürich.)

Sethi, D. N., P. K. Srivastava and P. Mahajan. 2002. Image analysis determination of microstructural parameters of snow and their correlation with snow's tensile strength. In Stevens, J. R., ed. International Snow Science Workshop 2002, 29 September-4 October 2002, Penticton, British Columbia. Proceedings. Victoria, B.C., B.C. Ministry of Transportation. Snow Avalanche Programs, 532-539. (http://www.fsavalanche.org/NAC/ techPages/articles/02_ISSW-Sethi.pdf.)

Szyszkowski, W. and P. G. Glockner. 1986. On a multiaxial constitutive law for ice. Mech. Mater., 5(1), 49-71. 